\title{
Marine ecology conditions at Weda Bay, North Maluku based on statistical analysis on distribution of recent foraminifera
}

\author{
Anis Kurniasih ${ }^{1}$, Septriono Hari Nugroho ${ }^{2}$, and Reddy Setyawan ${ }^{1}$ \\ ${ }^{1}$ Geological Engineering Diponegoro University, 50275 Semarang, Indonesia \\ ${ }^{2}$ Deep Sea Research Centres-LIPI, 16003 Bandung, Indonesia
}

\begin{abstract}
Analysis of foraminifera in geology, usually being used to find the age of rocks/ sediments and depositional environment. In this study, recent foraminifera was used not only to determine the sedimentary environment, but also to estimate the ecological condition of the water through a statistical approach.Analysis was performed quantitatively in 10 surface seabed sediment samples in Weda Bay North Maluku. The analysis includes dominance (Sympson Index), diversity and evenness (Shannon Index), and the ratio of planktonic - benthic. The results were shown in the plotting diagram of M-R-T (Miliolid-Rotalid-Textularid) to determine the depositional environment. Quantitative analysis was performed using Past software (paleontological version Statistic 1:29). The analysis result showed there was no domination of certain taxon with a moderate degree of evenness and stable communities and considerably a moderate diversity. The results of this analysis indicated that research area had a stable water conditions with the optimum level of carbonate content, oxygen supply, salinity, and temperature.The ratio of planktonic and benthic indicate the relative depth, which was deeper the water increased the percentage of planktonic foraminifera. Based on M-R-T diagram showed the distribution of sediment deposited on exposed carbonate (carbonate platform) environment with normal saline.
\end{abstract}

\section{Preliminary}

Analysis of the waters condition is very important because Indonesia is an archipelagic country that most of its territoryis covered by the sea. Ecological condition of a water body can become a reference about the usage of the waters for the fishing industry. The ecological condition of waters can be measured directly on water samples including the levels of carbonate, salinity, and temperature. However, this measurement is less precise to describe the ecological conditions in a long time scale. Ecological condition of waters can also be known by observing the organisms that live in the waters, which is to determine the correlation between the components of biotic and abiotic. One of the living organisms that is abundant in the sea is foraminifera.

This research was conducted by applying the method in seabed sediment samples taken from Weda Bay, North Maluku. The goal is to determine the recentecological condition of the water based on the distribution of foraminifera contained in the sediments. The benefit of the study is to determine the ecological conditions in a long time scale as the basis for the usage of the waters in fishing industry and so on.

Within the scope of geosciences, foraminifera are generally only used as a marker of age and depositional environment of rocks, but in this study,statistical methods was used in recent foraminiferacommunity in sediments to estimate the ecological condition of waters. Previous research on ecological analysis based on distribution of foraminifera had been done by [1] on the sediment surface in the Makassar Strait. The purpose of this study was to determine the distributional pattern of foraminifera, which include uniformity, diversity, and dominance of the individual as well as knowing the ecology of the waters at this time (Recent) based on the distribution of foraminifera contained in the sediments.

\section{Research method}

\subsection{Research site}

The research site was the territorial waters of Weda Bay, North Maluku (Fig.1). From all the sediment samples were taken, 10 of them performed a quantitative analysis of foraminifera, that was sample B2, B3, ST1, ST2, ST5, ST6, ST7, ST9, ST10, and ST11.

\subsection{Sampling method}

Sediment sampling was conducted by using a grab sampler. Sample of the sediment was weighed and then washed using a 100-mesh sieve with openings. Sample of the sediment was analyzed, mainly the foraminifera from 1 gram of sediment leftovers (washed residue). Thedistribution of foraminifera was quantified by the 
maximum number of 300 individuals.

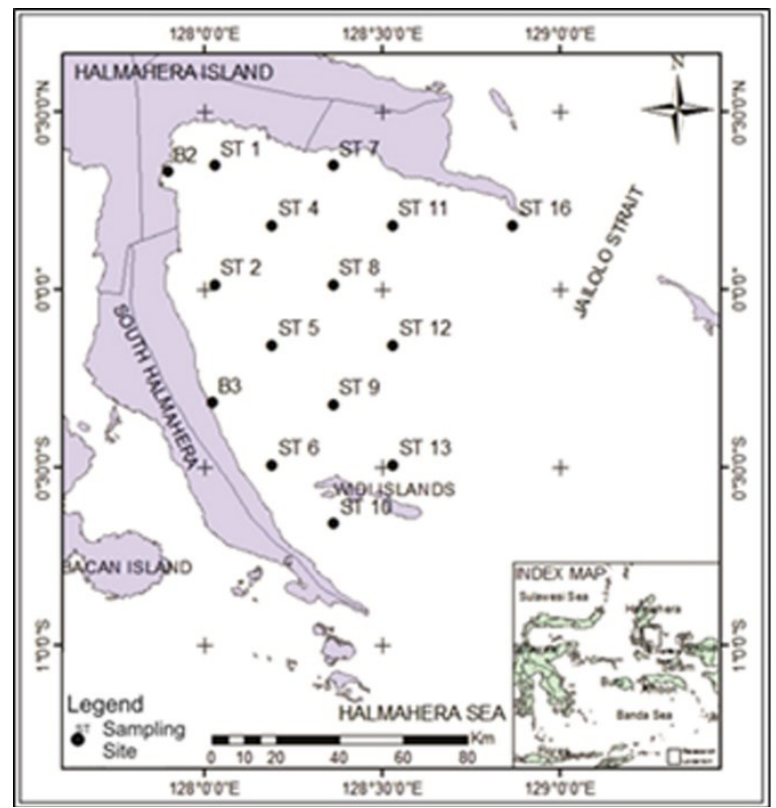

Fig. 1. Location of the study[2].

\subsection{Analysis of recent foraminifera}

\subsubsection{Qualitative and Quantitative analysis}

Qualitative analysis wasused to determine the taxonomic classification of foraminifera. Planktonic foraminifera taxonomy based on [3] andfor the benthic foraminifera was based on $[4,5,6]$. The taxonomic classification of foraminifera was the basis data to get the age and the depositional environment of sediment or rock samples.

Beside of qualitative analysis, this research used the quantitative analysis calculatedthe number and types of foraminifera that exist in each samples. The distribution of foraminifera was established in the distribution table, which then was used to conduct statistical analysis.

\subsubsection{Community Structure of Foraminifera}

Foraminifera community structure expresses in uniformity index (similarity), the diversity index (diversity), and dominance index. The variability or diversity is the number of species or taxon presents in a community. Diversity index in this study was performed using the index Shannon - Wiener [7], calculated as the formula below (1).

$$
H=-\sum \frac{n i}{n} \ln \left(\frac{n i}{n}\right)
$$

Where:

$$
\begin{aligned}
& \mathrm{H}=\text { Shannon-Wiener index } \\
& \mathrm{ni}=\text { Number of individuals of a taxon } \\
& \mathrm{n}=\text { total number of individuals }
\end{aligned}
$$

Diversity index criteria are divided into three categories [6]:

$\mathrm{H}<1=$ low diversity
$1<\mathrm{H}<3=$ moderate diversity

$\mathrm{H}>3=$ high diversity

In addition of using the index of Shannon - Wiener diversity can also be determined using the Fisherdiagram[8] via the alpha index calculation, as the following equation (2).

$$
\mathrm{S}=\alpha \ln (1+\mathrm{n} / \alpha)
$$

$$
\begin{aligned}
& \mathrm{S}=\text { the number of taxa } \\
& \mathrm{n}=\text { total number of individuals } \\
& \alpha=\text { total number of individuals }
\end{aligned}
$$

Uniformity value is the distributional pattern of each foraminiferataxonis equal or not, if the uniformity index value is relatively high the existence of any kind of foraminifera in the waters isunder equitable conditions. Uniformity index stated in Evenness Index (e), in accordance with the following formula (3) based on [7].

$$
e=\frac{H}{\log S}
$$

$$
\begin{aligned}
& \mathrm{e}=\text { evenness } / \text { uniformity index } \\
& \mathrm{H}=\text { index of Shannon }- \text { Wiener } \\
& \mathrm{S}=\text { the number of taxa }
\end{aligned}
$$

Value e $<0.3$ indicates uniformity is low, e $=0.3-0.6$ classified as moderate and $\mathrm{e}>0.6$ classified as high [7].

Dominance index is used to get information about taxon dominance in certain communities. Dominance index value ranging from $0-1$. If the dominance index value close to 1 then the community is dominated by certain taxon of foraminifera and if the dominance index close to 0 then no taxon dominates [7]. Here is the calculation formula dominance index created by [9] (4).

$$
D=\sum\left[\frac{n i}{n}\right]^{2}
$$

$\mathrm{D}=$ Dominance Index

$\mathrm{ni}=$ Number of individuals of a species I

$\mathrm{n}=$ total number of individuals

Community structure of foraminifera is one of the biotic parameters, which show the ecologic condition of waters, whether it is inhabitable by such organism, or not. This method is used to be a reliable method to determine the existence of disturbance in an area inhabited by a specific organism. The disturbance can occur by the cause of water pollution and or sedimentation.

\subsubsection{The individual presence ratio of planktonic and benthic foraminifera}

The individual presence ratio of planktonic and benthic foraminifera was used as an indicator of the depth of a water body [10]. 


\subsubsection{Triangular diagram}

Triangular diagram was arranged by the abundance of three types of foraminifera distinguished by the composition of foraminifera shell whether it wasagglutinate, porcelain, or hyaline (Fig.2). Triangular diagram parameter can be used as indicator of the carbonate, dissolved oxygen and salinity levels [11].

\section{Results and discussion}

\subsection{Foraminifera Community Structure}

Based on the quantitative analysis of 10 sediment samples taken from several locations in the study area (Fig. 1), obtained distribution of foraminifera which include uniformity, diversity, and their particular taxon dominates. Prior to the analysis, identification of each appearing taxon was performed and its population was counted. After that, all data was inserted in the program PAST [12] to determine the pattern of its spread (Table1).

Based on analysis of structural community in the foregoing discussion, it is known that the value of Shannon - Wiener diversity index of sample ST1, ST2, ST5, ST6, ST7, ST9, ST10, and ST 11ranged from 1-3, which means that the level of diversity is moderate. Fisher - Alpha index value of those samples show the level of diversity in the open ocean with normal salinity. This fits in with the results of the triangular diagram plotting showing the distributional pattern that characterizes the open sea environment with salinity, levels of carbonate and oxygen levels conditions were normal (normal marine continent).

Based on the dominance index value that is almost entirely close to 0 can be determine that there is no type of foraminifera that dominates from each community in the whole sample. Degree of uniformity or evenness of each total sample ranged from 0.3 to 0.6 , which means the level of uniformity moderate.

A conclusion from the analysis of the structural community is that the condition of the waters where the foraminifera were living was in optimum condition so that both planktonic and benthic organisms can thrive maximum. This can be seen from the absence of one type of foraminifera that dominates, meaning that each type of foraminifera can live and thrive. In addition, the level of diversity and uniformity were moderate, meaning that all types of foraminifera that live in the water has the same chance to thrive, or in other words, competition is low.Sample B2 and B3 were exceptional, because those samples were taken in transition between marine and terrestrial area, which not allowed foraminifera to live. Moreover, foraminifera that were occurred in those two samples might be derived from different environment. In other word, foraminifera that were contained in sample B2 and B3could not be used to indicate the structural community.

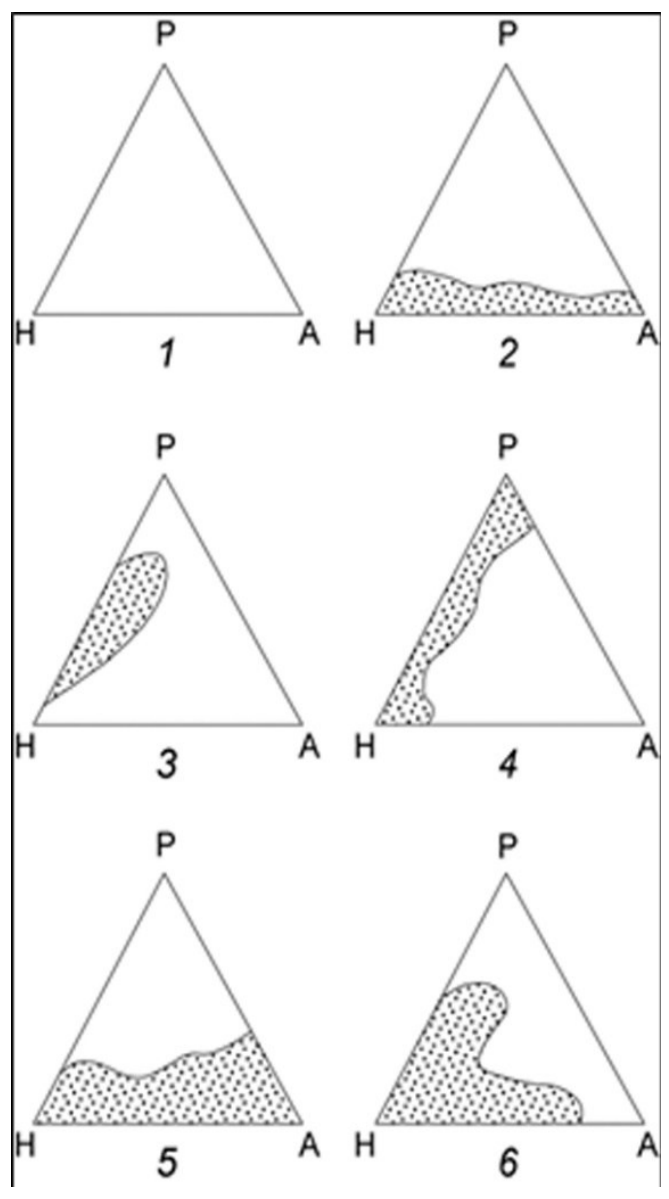

Fig. 2. Triangular diagram showing the abundance of foraminifera in several different environment [11], note: 1 . Fresh water; 2. Hypo saline lagoon; 3 . Normal marine lagoon and carbonate platform; 5. Shelf; 6 . Normal marine continental; P-porcelain; H-hyaline; A-agglutinated.

\subsection{The presence ratio of planktonic and Benthic Foraminifera}

Based on the ratio of planktonic and benthic foraminifera presence in the research area, nearly all samples had percentages of planktonic foraminifera presence far greater than benthic foraminifera, e.g. on the samples ST1, ST2, ST5, ST6, ST7, ST9, ST10, and ST 11. Planktonic foraminifera live by floating in the water and when it dies, the shell will be swept away. The higher the percentage of planktonic foraminifera presence indicates that the water allowed the shells of foraminifera to be deposited alongside other material. Thus, the place where the samplesweretakenpossibly has a low current or far away from the beach.

Meanwhile, there are two samples that have very lowpercentageof planktonic foraminiferaappearance compared to benthicforaminifera, the samples are B2 and B3. Based on observations in the microscope, sample B2 contains many pellets. Pellets are formed by 
Table 1.Calculation value of dominance index, uniformity Index, and diversity index using PAST software.

\begin{tabular}{|c|c|c|c|c|c|c|c|c|c|c|}
\hline & B2 & B3 & ST1 & ST2 & ST5 & ST6 & ST7 & ST9 & ST10 & ST11 \\
\hline Taxa S & 12 & 11 & 21 & 16 & 12 & 18 & 15 & 14 & 16 & 15 \\
\hline Individuals & 77 & 216 & 295 & 240 & 296 & 237 & 287 & 238 & 250 & 240 \\
\hline Dominance D & 0.1682 & 0.3791 & 0.1035 & 0.2378 & 0.2525 & 0.2165 & 0.1441 & 0.2858 & 0.3007 & 0.1801 \\
\hline Shannon H & 2.054 & 1.369 & 2.532 & 1.913 & 1.676 & 2.008 & 2.124 & 1.652 & 1.667 & 2.025 \\
\hline Simpson 1-D & 0.8318 & 0.6209 & 0.8965 & 0.7622 & 0.7475 & 0.7835 & 0.8559 & 0.7142 & 0.6993 & 0.8199 \\
\hline${\text { Evenness } e^{\wedge} \text { /S }}^{0.6499}$ & 0.3574 & 0.5988 & 0.4234 & 0.4452 & 0.414 & 0.5576 & 0.3725 & 0.3309 & 0.5048 \\
\hline Fisher alpha & 3.984 & 2.45 & 5.171 & 3.859 & 2.512 & 4.526 & 3.365 & 3.25 & 3.811 & 3.547 \\
\hline
\end{tabular}

chemical reactions that formed oval-shaped like material and contain many organic materials, probably derived from invertebrate organism's feces. Pellets are common in carbonate mud, as the sample B2 is based on granulometric analysis have mud dominant material. Water conditions that are dominated by mud do not allow foraminifera to live. The presence of foraminifera in the samples B2 likely derived from ocean currents that carry the shells of foraminifera from the sea towards the mainland. Meanwhile, sample B3 contained many plant particles, which indicates a high influence of land material so it does not allow foraminifera to live too. The presence of foraminifera in the samples B3 possibly formed during the transportation of sediment grain from the ocean.

The ratio of planktonic and benthicforaminifera individual presence can be seen in Table 2 .

Table 2. Appearance ratio of foraminifera planktonic and benthic.

\begin{tabular}{|c|c|c|c|c|c|}
\hline Code & Total Plankton & Total Benthic & Total Individual & percentage plankton & percentage benthic \\
\hline B2 & 21 & 56 & 77 & 27.3 & 72.7 \\
\hline B3 & 36 & 180 & 216 & 16.7 & 83.3 \\
\hline ST1 & 217 & 78 & 295 & 75.6 & 26.4 \\
\hline ST2 & 200 & 40 & 240 & 83.3 & 16.7 \\
\hline ST5 & 274 & 22 & 296 & 92.6 & 7.4 \\
\hline ST6 & 180 & 57 & 237 & 76 & 24 \\
\hline ST7 & 184 & 103 & 287 & 64.1 & 35.9 \\
\hline ST9 & 226 & 12 & 238 & 95 & 8.8 \\
\hline ST10 & 228 & 22 & 250 & 91.2 & 8.75 \\
\hline ST11 & 219 & 21 & 240 & 91.25 & \\
\hline
\end{tabular}

\subsection{Triangular diagram}

Triangular diagram was arranged based on the abundance of three types of foraminifera, distinguished by the composition of the shell wall, which is agglutinated, porcelain, and hyaline. The type of foraminifera that has an agglutinated shell wall are Bathysiphon sp. and Ammobaculites sp., while the type of foraminifera that has porcelain shell wall are Quinqueloculina sp. and Pyrgosp.Foraminifera which has a hyaline shell wall are Nodosaria sp., Elphidium sp., Uvigerinasp.,Cibicides sp., Obesopleustomella sp., Oridorsalisumbonatus, Bolivina sp., Bullimina sp., Lagena sp., Amphistegina sp., and Baculogypsinasp.The presence of these groups of foraminifera in each sample is plotted in the triangular diagram, as shown in Figure 3.

The resulting from plotting in triangular diagram of the entire sample (Fig.3) shows the distributional pattern that characterizes the open sea environment with salinity conditions, levels of carbonate and oxygen levels were normal (normal marine continent).

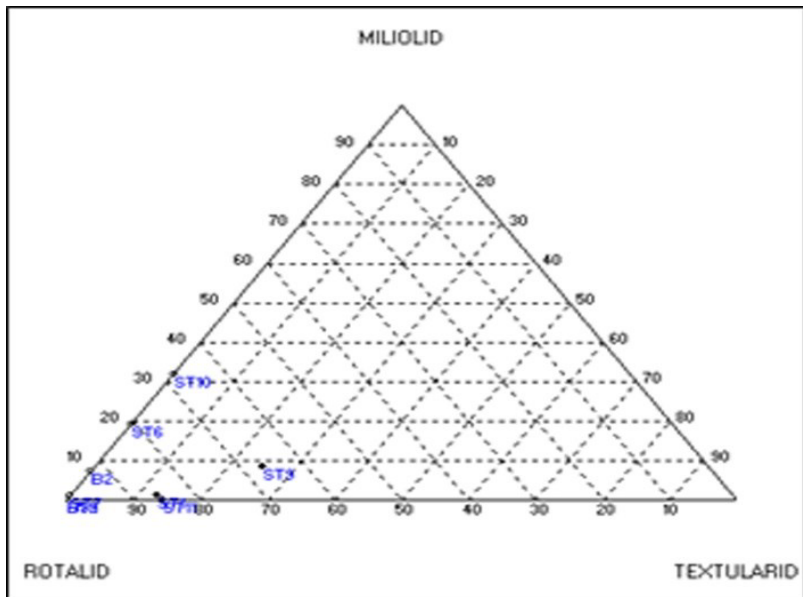

Fig. 3.Plotting a triangular diagram of all samples from the study area (Note: $\mathrm{P}=$ porcelain; $\mathrm{H}=$ hyaline; $\mathrm{A}=$ agglutinated).

\section{Conclusion}

Based on the stages and methods of research that has been done, it can be concluded as follows:

1. Based on analysis of structural community it is known that the value of Shannon - Wiener diversity index of all samples ranged from 1-3 which means that the level of diversity is moderate.

2. Fisher - Alpha index value shows the level of 
diversity in the open ocean with normal salinity.

3. The resulting from plotting in triangular diagram shows distributional pattern that characterizes the open sea environment with salinity conditions, levels of carbonate and oxygen levels were normal (normal marine continent).

4. Based on the dominance index value that is almost entirely close to 0 can be determine that there are no type of foraminifera that dominate from each community in the whole sample.

5. Degree of uniformity or evenness of each total sample ranged from 0.3 to 0.6 , which means the level of uniformity is moderate.

6. Based on the analysis of the community structure it can be seen that the condition of the waters where the foraminifera were lived in conditions of salinity, levels of carbonate and oxygen levels that optimum.

The authors thank to Abdul Basit, M.App.Sc as coordinator of the activities that have provided an opportunity for the retrieval and use of sediment samples from Weda Bay expedition in 2013 organized by UPT BKBL LIPI Ambon research funding sources DIPA 2013. The thanks also go to parties who have been willing to provide corrections, criticisms, suggestions and input, so that the research and writing ofthispapercan be resolved

\section{References}

1. S. M. Natsir, A. Firman, I. Riyantini, and I. Nurruhwati. J.IlmudanTek. KelautanTropis7,671-680 (2015).

2. S. H. Nugroho and A. Basit. J. IlmudanTek. KelautanTropis 6 No. 1,229-240 (2014).

3. J.A. Postuma. Manual of planktonik foraminifera. Amsterdam. Elsevier Publishing Company (1971) 252 420.

4. E. Boltovskoy. Marine Geo.26139-175 (1978).

5. A. R. Loeblich and H. N. Tappan. Foraminiferal genera and their classification. New York. Springer (1998).

6. F. P.C.M. van Morkhoven, W. A. Berggren, and A. S. Edward. Cenozoic cosmopolitan deep-water benthic foraminifera. Pau. Elf-Aquitaine (1986)

7. E. P. Odum. Fundamentals of ecology Third Edition. W.B. Philadelphia. Saunders Company (1971) 143 - 154.

8. J.W. Murray. Ecology and paleoecology of benthic foraminifera. Essex. Longman Harlow (1991).

9. E.H. Simpson. Measurement of diversity. Nature (1949) 163.

10. H. A. Armstrong and M. D. Brasier. Microfossils second edition. Cornwall. Blackwell Publishing (2005) 156

11. B. Valchev. 50 Years University of Mining and Geology "St.IvanRilski" Annual, Geology and Geophysics, Sofia, 46 (2003) 189-194.

12. Ø. Hammer, D.A.T. Harper, and P.D. Ryan. PAST: Paleontological statistic software package for education and data analysis.

(2004) 